\title{
Gold nanoparticles enhance cisplatin delivery and potentiate chemotherapy by decompressing colorectal cancer vessels
}

This article was published in the following Dove Press journal:

International Journal of Nanomedicine

\author{
Xiaoxu Zhaol,* \\ Jinghua $\operatorname{Pan}^{1, *}$ \\ Wei $\mathrm{Li}^{\prime}$ \\ Wende Yang' \\ Li Qin ${ }^{2}$ \\ Yunlong Pan' \\ 'Department of General Surgery, \\ The First Affiliated Hospital of Jinan \\ University, Guangzhou 510632. \\ China; ${ }^{2}$ Department of Histology and \\ Embryology, Medical School of Jinan \\ University, Guangzhou 510632, China \\ *These authors contributed equally \\ to this work
}

Background: Tumor vessels were persistently compressed by solid stress from tumor interstitial matrix, resulting in limited vessel perfusion and oxygen concentrations. Collagen within matrix participated in transmitting the solid stress to tumor vessels and limiting drug delivery.

Purpose: The objective of this study was to identify whether gold nanoparticles (AuNPs) were able to decompress colorectal cancer vessels and enhance vessel perfusion as well as drug delivery in colorectal cancer.

Methods: Colorectal cancer xenograft mice were treated with AuNPs or normal saline for 14 days. The cancer stromal collagen I level, cancer vessel perfusion, hypoxia of tumor were tested by histological examination. We also test the solid stress in the two groups. Furtherly, the effect and the drug delivery of combined using AuNPs and cisplatin were tested. The effect and the underlying mechanism of AuNPs on SW620 cells were tested by CCK8, flow cytometry, Western-blot and atomic force microscope.

Results: AuNPs were able to decrease the density of colorectal cancer associated fibroblasts (CAFs), to reduce the production of tumor stromal collagen I, and to diminish the expression of profibrotic signals, including CTGF, TGF- $\beta 1$ as well as VEGF in vivo and vitro via Akt signaling pathway. Consequently, AuNPs could alleviate solid stress in tumors, subsequently leading to enhanced vessel perfusion. Therefore, cisplatin as well as oxygen delivery to tumors were improved by AuNPs, which reduced hypoxia while sensitizing therapy of cisplatin in colorectal cancer model.

Conclusion: AuNPs were effective agents in enhancing cisplatin delivery and potentiating inhibiting tumor growth by decompressing colorectal cancer vessels.

Keywords: gold nanoparticles, colorectal cancer, vessel, decompression, solid stress, hypoxia

\section{Impact statement}

The organization and efficiency of vessel perfusion play a considerable role in the delivery of drug and oxygen to tumors. In this study, we found that gold nanoparticles (AuNPs) decreased colorectal cancer-associated fibroblast (CAF) density and reduced tumor stromal collagen I production, which were associated with decreased expression of profibrotic signals of TGF- $\beta 1$, connective tissue growth factor (CTGF), and vascular endothelial growth factor (VEGF). Consequently, AuNPs reduced solid stress and tumor vascular compression in tumors, which resulted in increasing vascular perfusion and drug and oxygen delivery to tumors, thereby potentiating chemotherapy in colorectal cancer model.

\section{Introduction}

The organization as well as efficiency of vessel perfusion plays a considerable role in the delivery of drug and oxygen to tumors. ${ }^{1,2}$ However, tumor vessels are abnormally
Correspondence: Yunlong Pan Department of General Surgery, The First Affiliated Hospital of Jinan University, No. 613, Huangpu Da Dao Xi, Tianhe District, Guangzhou 5I0632, China Email tpanyl@jnu.edu.cn 
compressed due to solid stress within the tumor microenvironment, resulting in the formation of hypoxic areas as well as hindering intratumoral drug delivery within the tumor microenvironment. ${ }^{3}$ The solid stress gradually initiates and progresses in tumors in response to the proliferation of cancer cells as well as stromal cells mediated by the cross talk of matrix molecules in tumor microenvironment. ${ }^{4}$ The tumor stroma reserves and further transmits the stress to tumor, ${ }^{5}$ leading to poor and inconsistent tissue perfusion attributed to distressed vessels, subsequently, causing hypoxia within the tumor microenvironment. Cisplatin, a well-known chemotherapeutic drug, has been used for the treatment of numerous human cancers including colorectal cancer. However, drug resistance and some side effects limited the drug's further use in anticancer therapy. In addition, the most prominent factor is the reduction in intracellular accumulation of cisplatin which leads many tumors to acquire the cisplatin resistance. ${ }^{6}$ Furthermore, combination therapies of cisplatin with other drugs have been highly considered to enhance drug delivery and overcome drug resistance. ${ }^{7}$ Some studies have showed that patients with poor tumor perfusion exhibit more insensitive chemotherapy response as well as shorter survival than those with satisfactory perfusion. ${ }^{8}$ Therefore, as the important component of tumor microenvironment, targeting tumor stroma and reducing solid stress of the tumor are considered a combination adjuvant therapy to enhance drug delivery to cancer tissues.

In spite of recognized contribution of matrix as well as stromal cells to solid stress, the precise mechanisms of the interplay between them to compress tumor vessels have not been clarified. Notably, a large number of cancer-associated fibroblasts (CAFs) and/or stellate cells (a type of stromal cell) produce as well as keep the extracellular matrix (ECM), which rely on host organ. Desmoplasia, mainly composed of hyaluronan, collagen fibers, and other ECM molecules, is prompted by the enhanced activity of these cells in tumors..$^{9,10}$ A clear elucidation of the mechanisms of tumor vessel compression would shed light on paradigms for promoting tumor perfusion.

Gold nanoparticles (AuNPs), versatile vehicles, are universally utilized in active and passive delivery of biomolecules to target organs. Drug-loaded nanoparticles are useful carriers to target tumors by the enhanced permeability and retention (EPR) effect, wherein nanoparticles of certain sizes tend to accumulate in tumors with highly permeable vessels. ${ }^{11}$ Our recent study has reported that AuNPs were able to enhance tumor vessel perfusion as well as oxygen supply to obtain optimal clinical outcomes in conjunction with recombinant human endostatin, elevation of tumor-selective delivery, induction of transient tumor vessel normalization, and reversal of epithelial-mesenchymal transition. ${ }^{12,13}$ In our previous researches, angiogenesis was reduced by monotherapy of AuNPs in hepatic cancer. ${ }^{14}$ In addition, migration as well as tube formation in human umbilical vein endothelial cells (HUVECs) via the Akt pathway triggered by vascular endothelial growth factor (VEGF)165 was inhibited by AuNPs. ${ }^{15,16}$

Although AuNPs have been previously classified as antiangiogenesis, in this study, we demonstrated that AuNPs could decrease colorectal CAF density, reduce the production of collagen I, and diminish the expression of profibrotic signals of TGF- $\beta 1$, connective tissue growth factor (CTGF) and VEGF. AuNPs were able to reduce solid stress, decompress colorectal cancer vessels, and increase cisplatin and oxygen delivery, ultimately improving chemotherapy response by the above-described mechanism. Therefore, solid stress can be alleviated by decreasing the expression of tumor matrix collagen as well as relevant agents to enhance tumor perfusion as well as chemosensitivity.

\section{Materials and methods Reagents and cells}

AuNPs were purchased from Shanghai Jie Ning Biotech Co., Ltd. (Shanghai, China). The average diameter of AuNPs was $15 \mathrm{~nm}$, which were dissolved in distilled water; then, they were kept in a light-resistant container at $4^{\circ} \mathrm{C}$. Cisplatin was purchased from Jiangsu Haosen Pharmaceutical Co., Ltd. (Lianyungang, China). The colorectal cancer cell line (SW620) was purchased from The Cell Bank of Type Culture Collection of Chinese Academy of Sciences (CBTCCCAS, Shanghai, China), which was maintained in DMEM containing 10\% FBS (Thermo Fisher Scientific, Waltham, MA, USA) in a $37^{\circ} \mathrm{C}$ incubator with $5 \% \mathrm{CO}_{2}$.

\section{Colorectal cancer xenograft models and treatment regimens}

The in vivo experiments in mice were approved by the Laboratory Animal Ethics Committee of Jinan University, which were conducted strictly following the relevant recommendations. Female Balb/c nude mice, 6-8 weeks old, were commercially obtained from the Beijing HFK Bioscience Co., Ltd. (Beijing, China), which were further kept in specific pathogen-free conditions. Briefly, SW620 cells $\left(5 \times 10^{6}\right)$ were subcutaneously injected into the right flank of the mice. Then, the tumor size was monitored using calipers, which was calculated with the following formula: volume $=($ length $\times$ width $\left.^{2} \times 0.523\right)$. Once the tumor volume reached $100 \mathrm{~mm}^{3}$, the animals were categorized into two groups $(\mathrm{N}=6$, each group) and treated with $1.0 \mathrm{mg} \cdot \mathrm{kg}^{-1}$ AuNPs or an equal amount of saline intravenously every other day for 14 days. The blood 
samples, liver, kidney, and the tumor tissues were collected and examined at day 14 .

\section{Enzyme-Linked Immuno Sorbent Assay (ELISA)}

Blood samples of xenograft nude mice were collected in test tubes and immediately centrifuged at 3,500 rpm for 15 minutes, followed by collection of plasma samples and storage at $-80^{\circ} \mathrm{C}$ before detection. SW620 cells were cultured in six-well plates with serum-free medium and treated with different concentrations of AuNPs (AuNPs1: $10 \mathrm{ng} / \mathrm{mL}$; AuNPs2: $25 \mathrm{ng} / \mathrm{mL}$; AuNPs3: $50 \mathrm{ng} / \mathrm{mL}$; AuNPs4: $100 \mathrm{ng} / \mathrm{mL}$ ). The cell culture medium was collected after treatment for 24 hours. ELISA Kit (Phoenix Pharmaceuticals, Burlingame, CA, USA) was used to assess the levels of IL-6, TNF- $\alpha$, TGF- $\beta 1$, CTGF, or VEGF in plasma following the protocols.

\section{Profibrotic marker detection of colorectal cancer}

At the termination of the animal experiment, $4 \mu \mathrm{m}$ sections of $4 \%$ paraformaldehyde-fixed paraffin-embedded tumor tissues were made. The slides were deparaffinized through a series of solutions (100\% xylene through $100 \%$ ethanol to $100 \%$ water), followed by incubation with alpha-smooth muscle actin ( $\alpha$-SMA; CAF; 1:200 dilution; Proteintech, Wuhan, China), active TGF- $\beta 1$ (1:500 dilution; Abcam, Cambridge, UK), CTGF (1:500 dilution; Abcam), or VEGF (1:250 dilution; Abcam) at $4{ }^{\circ} \mathrm{C}$ overnight. Then, tumor sections were treated with goat anti-rabbit $\operatorname{IgG}(\mathrm{H}+\mathrm{L})$ horseradish peroxidase $(1: 100$ dilution; Beyotime, Haimen, China) at $37^{\circ} \mathrm{C}$ for 40 minutes. Immunoreactivity was visualized with 3,3'-diaminobenzidine (DAB) using microscope (Leica DM6000B).

\section{Colorectal cancer stromal collagen I level}

For tumor stromal collagen I staining, the tumors were excised, frozen in optimal cutting temperature (OCT) compound (Sakura Finetek, Torrance, CA, USA), and then kept at $-80^{\circ} \mathrm{C}$. Later, cold acetone was used to fix the $5 \mu \mathrm{m}$ cryosections, followed by rehydration in PBS. Tissue sections were incubated with anti-collagen I antibody (1:200; Abcam) at $4{ }^{\circ} \mathrm{C}$ overnight, which were subsequently incubated with FITC-conjugated Affinipure Goat Anti-Rabbit IgG (1:200; Proteintech, Wuhan, China) for 1 hour in the dark. The staining was visualized using microscope (Leica DM6000B).

\section{Vessel perfusion of colorectal cancer}

For tumor vessel perfusion studies, a slow, intravenous injection ( $\sim 2$ minutes) of FITC-lectin (Sigma-Aldrich
Co., St Louis, MO, USA) was performed on nude mice at a concentration of $10 \mathrm{mg} \cdot \mathrm{kg}^{-1} 10$ minutes before the removal of tumors at the termination of the experiment. After dissection, tumor tissues were frozen in OCT compound and kept at $-80^{\circ} \mathrm{C}$. Then, cold acetone was used to fix the $5 \mu \mathrm{m}$ cryosections, followed by rehydration in PBS. Tissue sections were reacted with anti-CD31 antibody (1:500; Abcam), which were subsequently incubated with rhodamine goat anti-rat antibody (dilution 1:200; Proteintech, Wuhan, China) for 1 hour at dark. The staining was visualized using fluorescence microscope (Leica DM6000B). The proportion of tumor vessel perfusion was defined as the ratio of the lectin + area to CD31+ area using Image $\mathrm{J}$ software.

\section{Detection of colorectal cancer hypoxia}

To evaluate the hypoxia in tumor, an intraperitoneal injection of pimonidazole at a dose of $60 \mathrm{mg} \cdot \mathrm{kg}^{-1}$ was carried out in nude mice 1 hour before tumor excision. After fixation of the tumor tissues, sections were made as described earlier. Hypoxyprobe-1 Plus kit (HPI Inc, Burlington, MA, USA) was purchased for pimonidazole staining following the manufacturer's protocol. Hypoxyprobe-1 adducts were examined with an affinity-purified rabbit IgG polyclonal antibody conjugated with horseradish peroxidase $(1: 100$; Beyotime).

\section{Histologic image analysis}

We randomly chose six fields at $\times 200$ magnification in each slide under a fluorescence microscope (Leica DM6000B). To determine the proportion of vessel perfusion, the number of vessels counted by this program with the colocalization of lectin and CD31 staining was divided by the number of vessels counted with CD31 staining. Images of collagen I, pimonidazole, $\alpha-S M A$ (CAF), TGF- $\beta 1$-, CTGF-, or VEGFstained sections were evaluated according to the area proportion of positive staining using Image $\mathrm{J}$ software. In the assessment of all tumors, identical analysis settings and thresholds were employed.

\section{Colorectal cancer: solid stress}

The tumor-opening technique was employed to detect solid stress. ${ }^{3}$ The nude mice were anesthetized at the termination of the experiment, followed by tumor excision, whose three dimensions were determined after washing with Hank's balanced salt solution (HBSS). Specifically, a scalpel was used to cut the tumor alongside the longest axis, to a depth of $80 \%$ of the shortest axis. Then, the tumors were placed in HBSS again for 10 minutes to eliminate the transient, 
poro-elastic responses. The opening area triggered by the cut was subsequently detected at the middle of the cut, which was located on the tumor surface. Solid stress was calculated as the ratio of the size of the opening to the size of the dimension perpendicular to the cut.

\section{Colorectal cancer: cisplatin delivery and tumor growth}

Nude mice bearing ectopic SW620 cells were split into four groups ( $\mathrm{N}=6$ per group) when the tumor volume reached $100 \mathrm{~mm}^{3}$ post inoculation. The animals were intravenously injected with $1.0 \mathrm{mg} \cdot \mathrm{kg}^{-1}$ AuNPs, $5.0 \mathrm{mg} \cdot \mathrm{kg}^{-1}$ cisplatin, a combination of $1.0 \mathrm{mg} \cdot \mathrm{kg}^{-1}$ AuNPs and $5.0 \mathrm{mg} \cdot \mathrm{kg}^{-1}$ cisplatin, or an equal volume of saline every other day for 14 days. At the termination of the experiment, animals were retroorbitally administered with $10.0 \mathrm{mg} \cdot \mathrm{kg}^{-1}$ cisplatin, half an hour before the removal of tumors and organs. Then, the tumors were extracted, followed by the measurement of the volume by calipers. After dabbing off the excessive blood, the tumor tissues were snap-frozen in liquid nitrogen for analysis. HPLC (Agilent Technologies, Santa Clara, CA, USA) on a COSMOSIL C18 column $(250 \times 4.5 \mathrm{~mm}, 5 \mu \mathrm{m}$; Shimadzu, Tokyo, Japan) was employed to determine the cisplatin concentration extracted from the tumor tissues. Agilent ChemStation software was utilized to analyze all the acquired responses.

\section{CCK-8 assay}

$5 \times 10^{3} \mathrm{SW} 620$ cells/well were inoculated in 96-well plates and treated with different concentrations of AuNPs for 24 hours (AuNPs1: 10 ng/mL; AuNPs2: 25 ng/mL; AuNPs3: $50 \mathrm{ng} / \mathrm{mL}$; AuNPs4: $100 \mathrm{ng} / \mathrm{mL})$. Twenty microliters of CCK-8 solution was added to each culture well and incubated for 4 hours. After shaking at a constant temperature for 10 minutes, the absorbance (OD) of each group was measured with a multifunctional microplate reader (measured at a wavelength of $540 \mathrm{~nm}$ ). The proliferation of sw620 cells was observed. Cell proliferation rate $(\%)=($ experimental group OD value/control group OD value) $\times 100 \%$.

\section{Western blot}

SW620 cells $\left(1 \times 10^{6}\right)$ were planked in six-well plates with DMEM $+10 \%$ FBS. After 24 hours, the medium was changed to serum-free medium and then incubated with AuNPs (50 ng/mL), VEGF165, or VEGF121 (20 ng/mL; catalog no: 68-8784-63 and RP-8646; Thermo Fisher Scientific) for 24 hours. The cells were washed with ice-cold PBS and lysed in lysis buffer. The lysis $(50 \mu \mathrm{g})$ was separated by
SDS-PAGE and transferred to polyvinylidene difluoride (PVDF) membranes. TGF- $\beta 1$, CTGF, VEGF, and phosphorylated Akt were analyzed using a TGF- $\beta$ Rabbit mAb (no 3709), Rabbit CTGF mAb (no 86641), Rabbit VEGF antibody (no 2463), Akt mAb (no 4691), and Akt rabbit phospho-Akt mAb (no 4060; Cell Signaling, Beverly, MA, USA). The GAPDH (no 5174) was used as a loading control. The anti-rabbit peroxidase-conjugated secondary antibody was detected using enhanced chemiluminescence reagents.

\section{Apoptosis analysis by flow cytometry}

SW620 cells were seeded into six-well plates followed by the addition of different concentrations of AuNPs for 24 hours. Then, cells were collected and washed twice with PBS. Annexin V-APC/7-amino-actinomycin D (AAD) Apoptosis Detection Kit (KeyGEN Biotechnology, Nanjing, China) was employed to determine apoptotic cells by flow cytometry. In brief, $5 \mu \mathrm{L} 7$-ADD and $50 \mu \mathrm{L}$ binding buffers were added for cellular staining in dark and room temperature conditions. Then, $450 \mu \mathrm{L}$ binding buffers and $1 \mu \mathrm{L}$ Annexin V-PE were used for staining in the same conditions. The results were analyzed via flow cytometry (ACEA NovoCyte ${ }^{\circledR}$; Biosciences, Inc., San Diego, CA, USA) and followed by NovoExpress 1.2.4 software (ACEA Biosciences, Inc.).

\section{Atomic force microscope (AFM) analysis}

SW620 cells $\left(1 \times 10^{4}\right)$ were planked on $22 \times 22 \mathrm{~mm}$ coverslips in six-well plates and were treated with different concentrations of AuNPs for 24 hours. Then, the coverslip was fixed by $4 \%$ paraformaldehyde and waited for natural drying. The image was scanned with an AFM at room temperature. The experiment used a $100 \mu \mathrm{m}$ scanner, contact mode, and silicon probe, and the resonance frequency was $250-320 \mathrm{~Hz}$. All the images were analyzed via Proscan Image Processing Software Version 2.1.

\section{Statistical analyses}

The experiments were performed in triplicates, and the data were shown as mean \pm standard error (SE). GraphPad Prism was utilized in all statistical analyses (version 5.0; GraphPad Software, Inc., La Jolla, CA, USA). The two-tailed Student's $t$-test was used to determine the statistical significance of the difference between two groups. One-way ANOVA was used in comparisons of different groups, followed by Bonferroni's post hoc test for parametric data and Kruskal-Wallis test followed by a Dunn's multiple comparison test. A $P$-value of $<0.05$ was considered to be statistically significant $(* P<0.05, * * P<0.01$, and $* * * P<0.001)$. 


\section{Results}

\section{Characterization of AuNPs}

AuNPs were spherical granules with good dispersity as well as consistent size, as illustrated by transmission electron microscopy (TEM) images (Figure 1A and B). In addition, the surface plasmon resonance (SPR) absorption peaks of AuNPs was at around $517 \mathrm{~nm}$, indicated by the ultraviolet (UV)-visible spectrum (Figure 1C). The fluid particle size, zeta potential, and $\mathrm{pH}$ value of AuNPs are summarized in Table 1.

\section{AuNPs reduce colorectal cancer stromal collagen I}

Collagen I is widely involved in tumor vessel compression, which subsequently triggers blood flow reduction as well as considerable unperfused tumor areas. Moreover, previous studies have demonstrated the facilitation of the stiffening and reorganization of the collagen I via CAFs in tumor cell invasion. In this study, the rapid inhibition of tumor expression of collagen I by AuNPs was verified $(P<0.001$; Figure $2 \mathrm{~A}$ and $\mathrm{B}$ ), indicating that collagen I was degraded or destabilized by AuNPs.

\section{AuNPs reduce colorectal cancer stromal fibrosis signaling}

To determine the tumor stromal fibrosis signaling after treatment with AuNPs or saline, ELISA kit and immunohistochemistry (IHC) were employed to explore how AuNPs affected TGF- $\beta 1$, CTGF, or VEGF expressions in colorectal cancer model. As a result, AuNPs reduced plasma levels of TGF- $\beta 1$, CTGF as well as VEGF (all $P<0.001$; Figure $3 \mathrm{~A}-\mathrm{C}$ ) in nude mice. Moreover, the reduced tumor expressions of TGF- $\beta 1, \mathrm{CTGF}$, and VEGF by AuNPs were determined through immunohistochemistry assessment (*** $P<0.001$; Figure 3D).

As described earlier, the rapid reduction in tumor stromal collagen I level by AuNPs indicated that collagen I was degraded or destabilized by AuNPs via an undefined mechanism besides blocking the matrix production through TGF- $\beta 1$. CTGF is a matricellular protein, involved in stabilization of transient fibrosis triggered by TGF- $\beta 1$ activity, ${ }^{17,18}$ implying that AuNPs were able to destabilize collagen I through the inhibition of CTGF expression. In addition, the expressions of CTGF and TGF- $\beta 1$ were decreased by AuNPs in colorectal cancer. Thus, besides the blocking in TGF- $\beta 1$ pathway, AuNPs were likely to decrease stromal collagen I expression partially by destabilization in the existing collagen I through CTGF. VEGF, a homodimeric glycoprotein, plays a core role in angiogenesis by the regulation of the tumor neovascularization as well as permeability of blood vessels..$^{19,20}$ Besides, previous studies have revealed an elevated VEGF level in fibrosis and inflammatory diseases, including rheumatoid disease, ocular disease, and Crohn's disease. ${ }^{21,22}$ Our result showed that AuNPs also reduced plasma VEGF concentrations in mice. Taken together, these effects contributed to the reduced level of collagen I in colorectal cancer.

\section{AuNPs decrease colorectal CAF density in tumors}

Next, we decided to assess whether colorectal CAF density could be reduced by AuNPs as well. CAFs are typically identified by $\alpha$-SMA, a myocyte marker ${ }^{23,24}$ and other agents, such as fibroblast-specific protein (FSP-1). CAFs comprise of, as high as, $80 \%$ of cells in the entire tumor mass, such as pancreatic cancer. Functionally, CAFs are deeply involved in cancer development, progression, and metastasis. ${ }^{25}$ In our study,
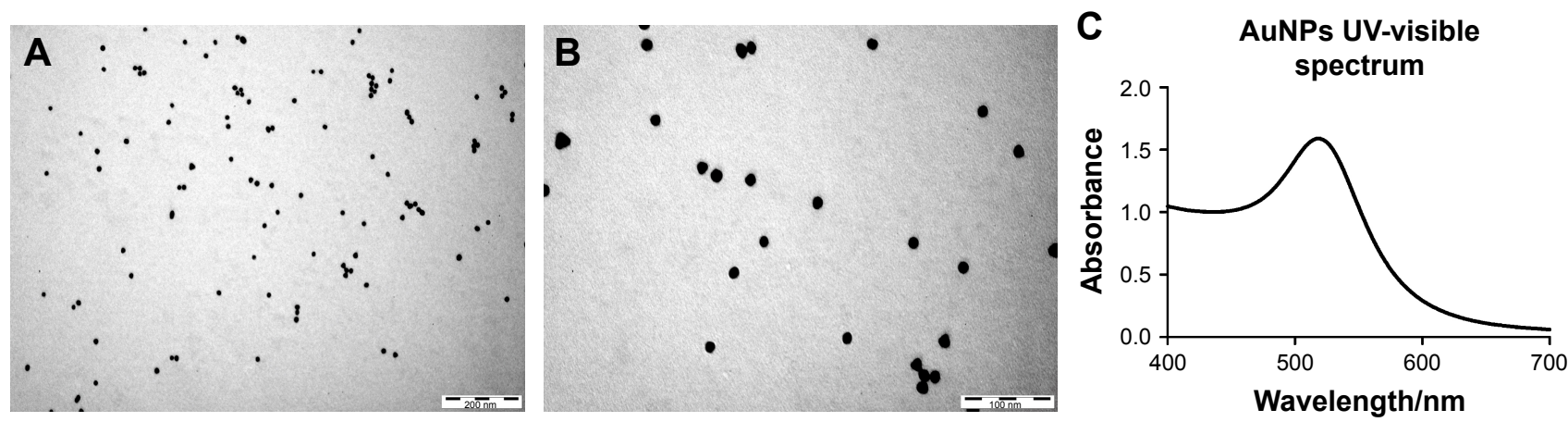

Figure I Characterization of AuNPs.

Notes: (A, B) FETEM images reveal discrete particles of AuNPs. Scale bar: $200 \mathrm{~nm}$ and $100 \mathrm{~nm}$. (C) UV-visible spectra of AuNPs. The UV-visible spectra of I5 nm AuNPs were characterized by surface plasma resonance absorption peaks at approximately $517 \mathrm{~nm}$.

Abbreviations: AuNPs, gold nanoparticles; FETEM, field-emission transmission electron microscopy; UV, ultraviolet. 
Table I The parameters of AuNPs

\begin{tabular}{l|l}
\hline Parameters & AuNPs \\
\hline Fluid particle size $(\mathrm{nm})$ & $14.34 \pm 0.75$ \\
Zeta potential $(\mathrm{mV})$ & $-21.19 \pm 0.64$ \\
$\mathrm{pH}$ value & 8.0 \\
\hline
\end{tabular}

Abbreviation: AuNPs, gold nanoparticles.

the density of $\alpha$-SMA-positive cells was decreased by the administration of AuNPs in colorectal cancer $(* * P=0.0032$; Figure $4 \mathrm{~A}$ and $\mathrm{B}$ ), indicating a declined activity of CAFs. Therefore, AuNPs were likely to inhibit the activation of fibroblasts to $\alpha$-SMA + CAF phenotype, thereby resulting in reduced CAF density.

\section{AuNPs reduce solid stress in colorectal cancer}

Next, the role of AuNPs on solid stress in colorectal cancer was evaluated. Solid stress is mainly generated by fibroblast stress and kept as strain energy in the ECM. Subsequently, the cooperation of hyaluronan as well as collagen transmitted the strain energy to compress blood vessels, ultimately causing inconsistent, unsatisfactory tissue perfusion as well as hypoxia in the tumor microenvironment. Overall, these factors facilitated tumor progression. ${ }^{26,27}$ Solid stress in tumor was determined by recently established technique in this study, ${ }^{3}$ which showed that AuNPs reduced solid stress in colorectal cancer $(* * * P=0.0002$; Figure 5$)$. Therefore, AuNPs were likely to decompress vessels by relieving solid stress due to its antimatrix roles.

\section{AuNPs increase colorectal cancer vessel perfusion}

From the abovementioned results in the desmoplastic tumor model, we cautiously speculated that the decreased solid stress by AuNPs might enhance vessel perfusion. As a result, the tumors were burdened with the loss of sufficient blood perfusion, which was indicated that blood perfusion was only detected in $23 \%$ of vessels in colorectal cancer ( ${ }^{* *} P=0.0026$; Figure 6A). After the administration of AuNPs, the proportion of perfused vessels significantly rose to, as high as, $43 \%$, which implicated that vascular decompression was at least part of the mechanism. ${ }^{6}$ Thus, AuNPs were capable of enhancing blood perfusion in cancers through the reduction in solid stress.

\section{AuNPs enhance oxygen and colorectal cancer cisplatin delivery}

In view of the governing role of vessel perfusion in oxygen as well as drug delivery, we first detected tumor oxygenation using pimonidazole staining. Consequently, oxygen concentrations were maintained in SW620 cells after the administration of AuNPs, while a typical growth-dependent decline in oxygenation was observed in the control group $(* * * P<0.001$; Figure 6B). Therefore, the reduction in tumor solid stress via "microenvironmental normalization" mediated by AuNPs promoted the delivery of oxygen as well as drugs. Moreover, tumors excised from mice with the treatment of AuNPs harbored significantly smaller necrotic regions of the entire tumor mass, as indicated by immunohistochemistry staining ( $* * * P<0.001$, Figure $6 \mathrm{C})$. Next, to determine whether AuNPs affected cisplatin delivery to cancers, the cumulative degrees of small molecule chemotherapeutic cisplatin in the tumor, liver, and kidney were assessed by HPLC. The results showed that AuNPs improved cisplatin delivery to SW620 tumor $(* * P=0.0023$; Figure $6 \mathrm{D})$, but not to liver $(P=0.2365$; Figure $6 \mathrm{E})$ or kidney $(P=0.3314$; Figure 6F), which implicated that only tumor tissues were targeted by the delivery-enhancing strategy mainly due to
A

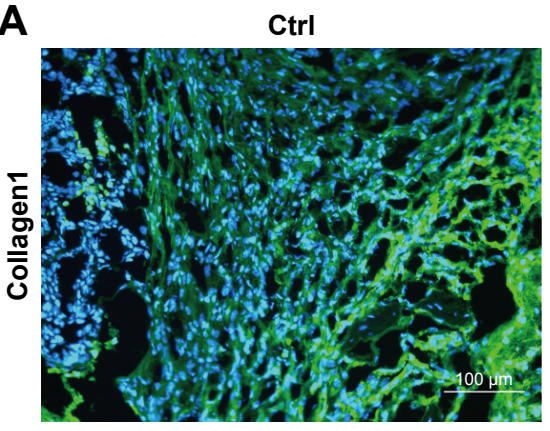

AuNPs

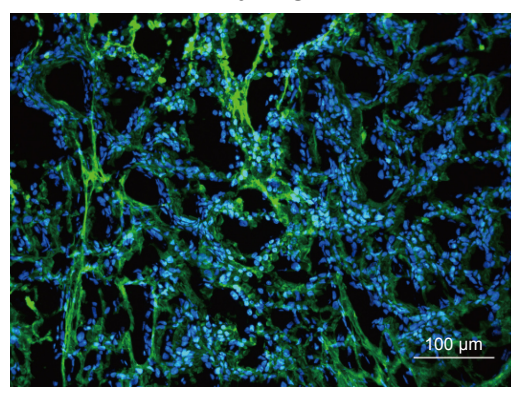

B

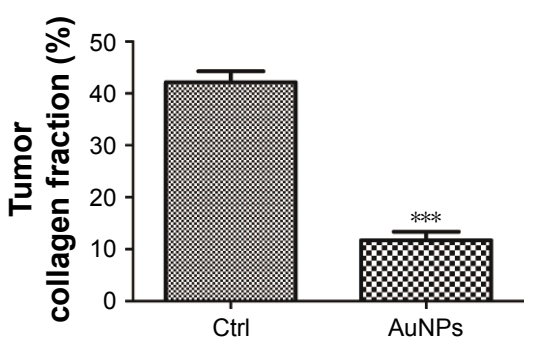

Figure 2 AuNPs decreased stromal collagen I level in SW620 tumor. (A) Immunofluorescence images of the effect of AuNPs on stromal collagen I (green) level in SW620 tumor. Scale bar, $100 \mu \mathrm{m}$. (B) Quantification of tumor collagen I level after the administration of AuNPs. AuNPs reduced the proportion of collagen I-positive region in SW620 tumor $(n=6, * * * P<0.00$ I, Student's t-test).

Note: Error bars indicate SEM.

Abbreviations: AuNPs, gold nanoparticles; Ctrl, control; SEM, standard error of the mean. 
A

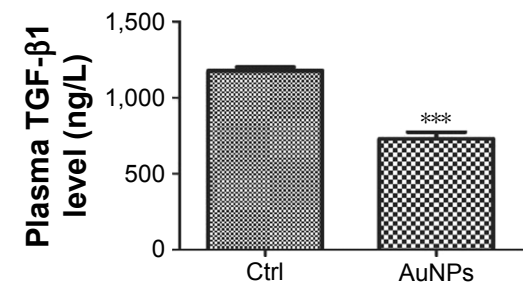

D
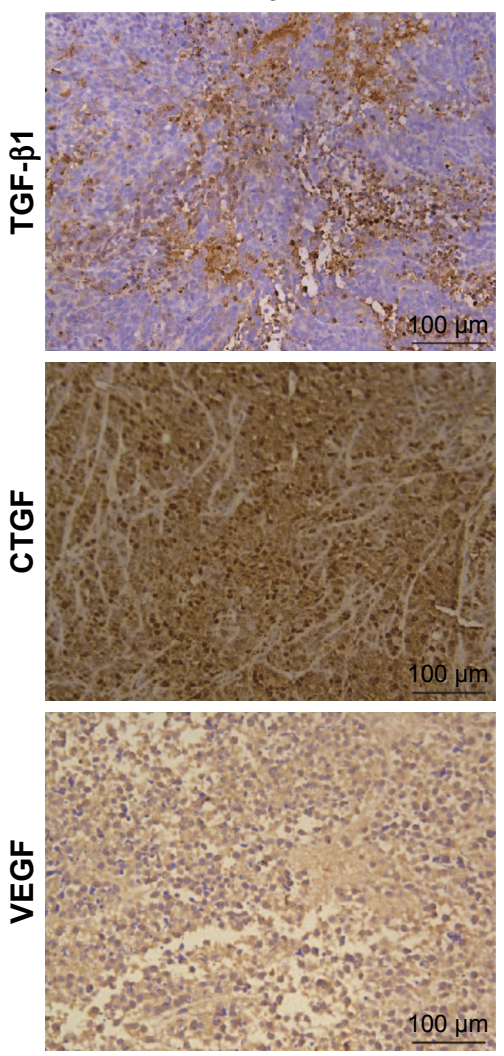

B

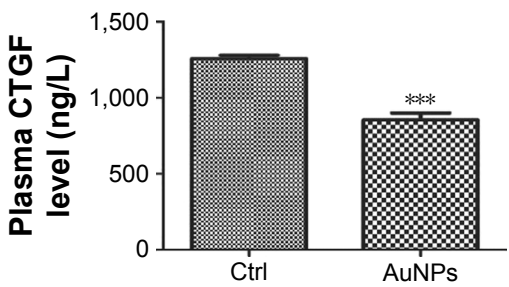

AuNPs
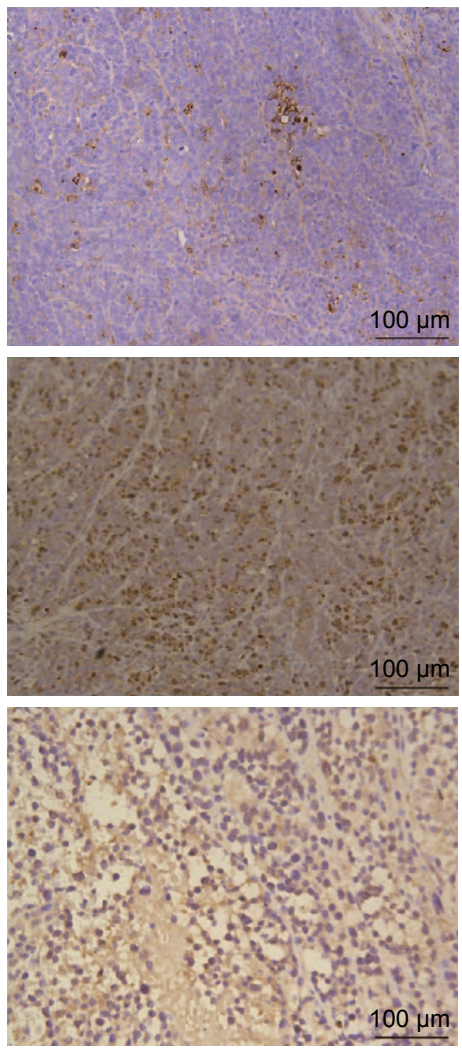

C

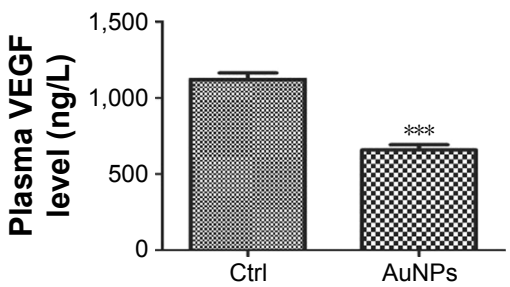

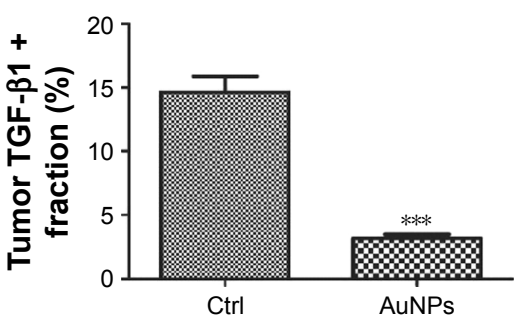
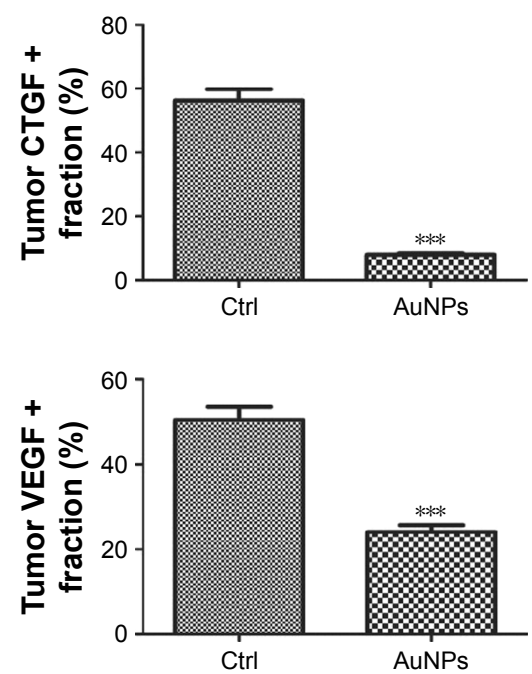

Figure 3 AuNPs decreased profibrotic cytokine levels in plasma and SW620 tumor.

Notes: (A) Plasma level of TGF- $\beta$ I was decreased after treatment with AuNPs ( $n=6, * * * P<0.00 \mathrm{I})$. (B) Plasma level of CTGF was decreased ( $\mathrm{n}=6$, $* * * P<0.00 \mathrm{I}$ ). (C) The plasma level of VEGF was reduced after the administration of AuNPs ( $n=6$, *** $P<0.00 I)$. (D) Histologic images of the effect of AuNPs on TGF- $\beta$ I, CTGF, and VEGF expression in SW620 tumor ( $\mathrm{n}=6)$. Scale bar, $100 \mu \mathrm{m}$. AuNPs decreased the expression of TGF- $\beta$ I, CTGF, and VEGF (***all $P<0.00 \mathrm{I})$ in SW620 tumor. Student's $t$-test was used for analysis, and error bars indicate SEM.

Abbreviations: AuNPs, gold nanoparticles; CTGF, connective tissue growth factor; Ctrl, control; SEM, standard error of the mean; VEGF, vascular endothelial growth factor.

A

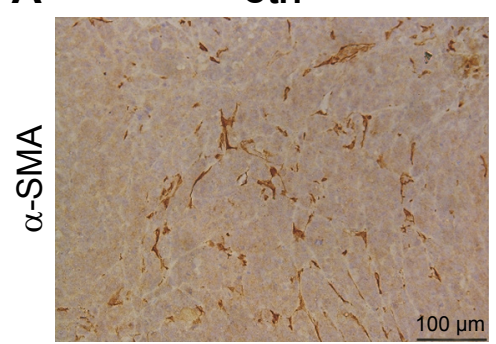

AuNPs

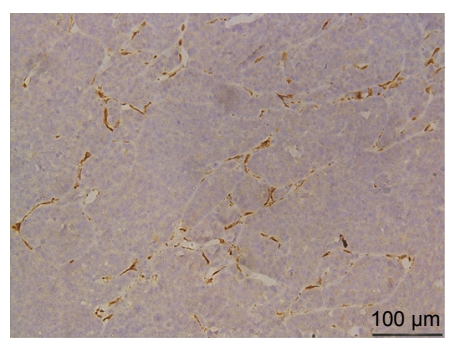

B

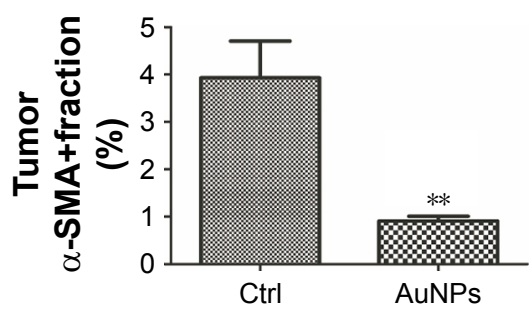

Figure 4 AuNPs reduced $\alpha$-SMA-positive CAF density in SW620 tumor.

Notes: (A) Histologic imaging illustrating the effect of AuNPs on the level of $\alpha$-SMA-positive CAFs (brown) in tumor tissues. Scale bar, I00 $\mu$ m. (B) Immunohistochemical analysis showed that the administration of AuNPs diminished the $\alpha$-SMA-positive CAF density in SW620 tumor ( $n=6$, **P=0.0032, Student's $t$-test). Error bars indicate SEM. Abbreviations: AuNPs, gold nanoparticles; $\alpha$-SMA; alpha-smooth muscle actin; CAFs, cancer-associated fibroblasts; Ctrl, control; SEM, standard error of the mean. 


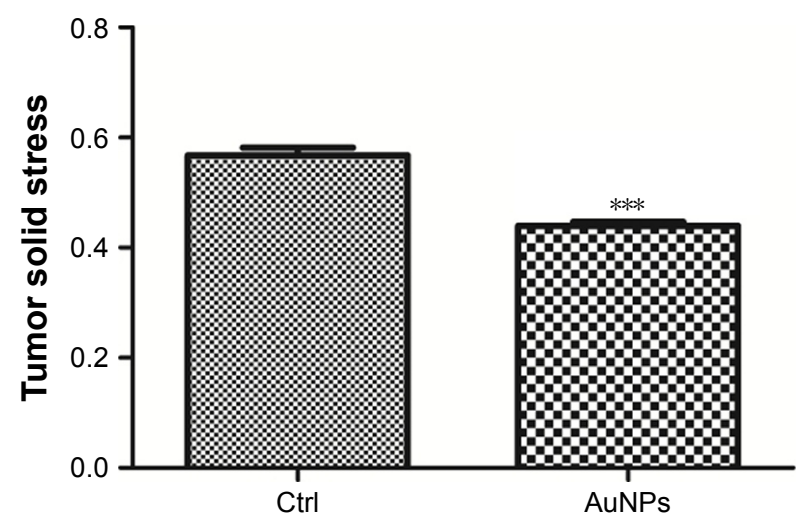

Figure 5 AuNPs reduced solid stress in SW620 tumor.

Notes: An ex vivo technology was employed to evaluate tumor solid stress by detecting the degree of tumor tissue relaxation (tumor opening relative to tumor diameter) after a stress-releasing incision, with larger openings indicating higher stress. Through its antimatrix effects, AuNPs decreased solid stress in SW620 tumor $(n=6, * * * P=0.0002$, Student's $t$-test). Error bars indicate SEM.

Abbreviations: AuNPs, gold nanoparticles; Ctrl, control; SEM, standard error of the mean.

the specific accumulation of solid stress in tumor tissues. Therefore, the administration of AuNPs could temporarily promote blood perfusion, while relieving hypoxia as well as necrosis in cancers.

\section{AuNPs potentiate the efficacy of cisplatin}

To further study the roles of AuNPs on delivery, a combined administration of AuNPs and cisplatin was conducted on SW620 tumors. Both cisplatin monotherapy and cisplatin combined with AuNPs significantly inhibited tumor growth (Figure 7A). Nevertheless, a more obvious reduced effect was observed in mice with a combined administration of AuNPs and cisplatin. The body weight of four groups had no significant difference after treatment for $0-14$ days (Figure 7B). The histopathological examination of major organs also showed no significant injury after AuNPs or combined administration for 14 days (Figure 7C). The serum IL-6 and TNF- $\alpha$ in the cisplatin and combination group were significantly increased comparing with the control and AuNPs group after treatment for 1, 5, and 10 days. However, there was no significant difference in serum IL- 6 and TNF- $\alpha$ levels between the cisplatin and combination group (Figure 7D and E). In line with the abovementioned results, HPLC analysis detected high concentrations of cisplatin in tumors after combined therapy, both of which indicated that the delivery efficacy of cisplatin was promoted by tumor vessel decompression after the administration of AuNPs. Collectively, AuNPs were able to enhance the therapeutic outcomes of cisplatin via antimatrix effects and did not increase the toxicity when combined with cisplatin.

\section{AuNPs decrease the production of TGF- $\beta$ I, CTGF, and VEGF via Akt- dependent pathway}

To further clarify the underlying mechanism of AuNPs on colorectal cancer, we investigated the effect of AuNPs on SW620 cells in vitro. First, there was no significant difference of viability and apoptosis rate of SW620 cells after treatment with different concentrations of AuNPs (Figure 8A-C), indicating that the effect of AuNPs on tumor growth was not mediated by the inhibition of cell proliferation and induction of cell apoptosis. In addition, Western blot showed that the expressions of TGF- $\beta 1, \mathrm{CTGF}$, and VEGF were decreased in the AuNP treatment groups compared with the control group (Figure 8D). As reported in our previous study,,$^{14}$ AuNPs could inhibit the binding of VEGF165 to VEGFR2 via the heparin-binding capacity of AuNPs, and the VEGFR2/PI3K/ Akt axis played an important role in regulating the production of TGF- $\beta 1$, CTGF, and VEGF. ${ }^{28-30}$ Hence, our results further confirmed that the AuNPs could inhibit VEGF165-induced, but not VEGF121-induced, Akt phosphorylation in colorectal cancer cells (Figure 8E). Finally, we detected the secretions of TGF- $\beta 1$, CTGF, and VEGF in culture medium of SW620 cells. After AuNP treatment with different concentrations, the levels of TGF- $\beta 1, C T G F$, and VEGF in culture medium were significantly decreased compared with the control group $(P<0.001$; Figure $8 \mathrm{~F}-\mathrm{H})$. Therefore, our study indicated that AuNPs can reduce TGF- $\beta 1$, CTGF, and VEGF production via Akt-dependent pathway.

\section{Cellular uptake and internalization of AuNPs were visualized by AFM}

Finally, we further measured the cellular uptake and internalization of AuNPs on colorectal cancer cells. The AFM was used to analyze the effects of AuNPs on the morphology and surface ultrastructure of SW620 cells. As shown in Figure 9, the surface and the cytoplasm of SW620 cells were intact and full in the control group. After AuNP treatment with $10.0 \mathrm{ng} / \mathrm{mL}$ or $25.0 \mathrm{ng} / \mathrm{mL}$, membrane invagination and increasing of surface pore were found in SW620 cells. These results indicated that colorectal cancer cells can uptake and internalize AuNPs.

\section{Discussion}

In this study, the complicated effect of tumor matrix on impeding drug delivery has been comprehensively assessed. The excessive production of ECM by cancer cells, CAF, and other stromal cells serves as structural support of the tumor microenvironment, and there are dynamic molecular 
A

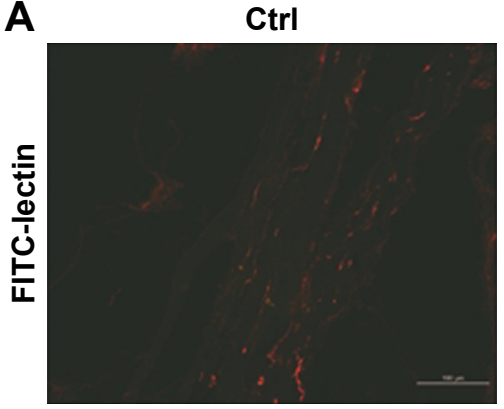

B

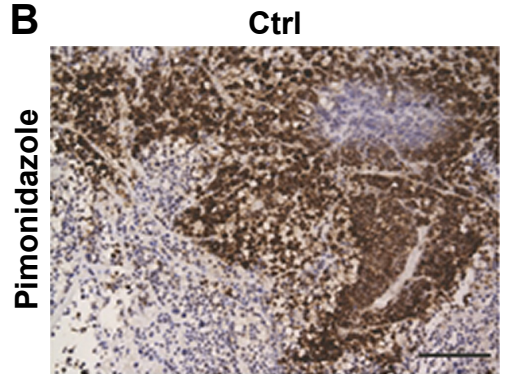

C

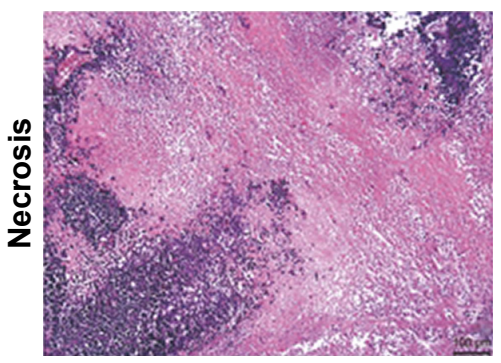

D

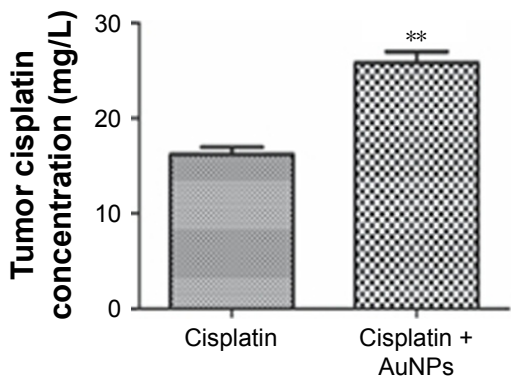

AuNPs

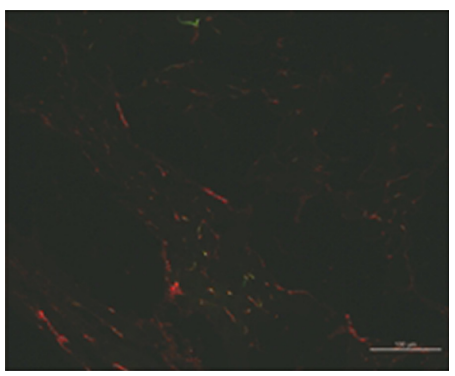

AuNPs

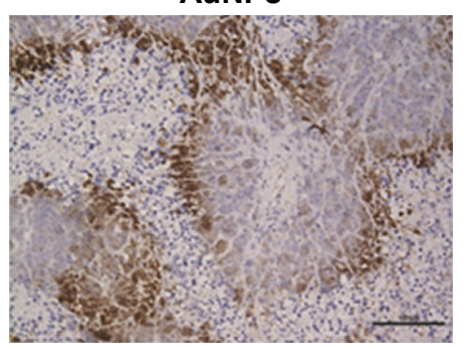

AuNPs

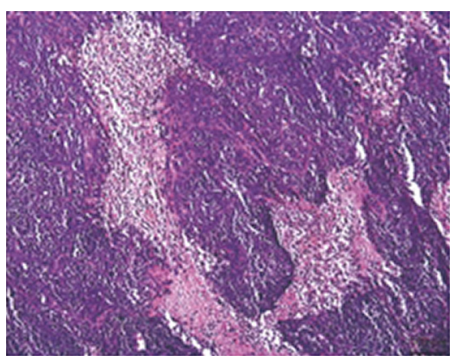

E

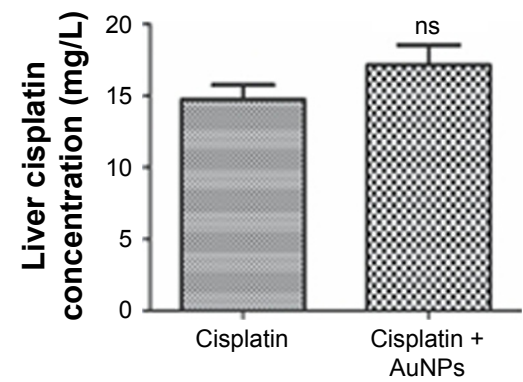

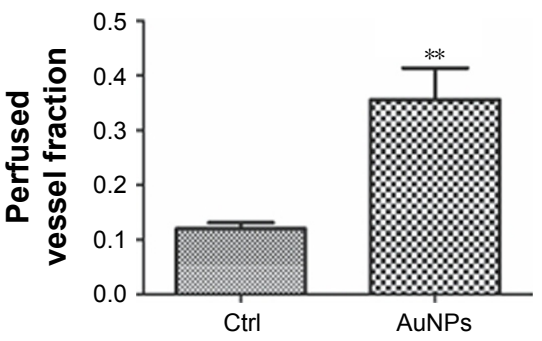
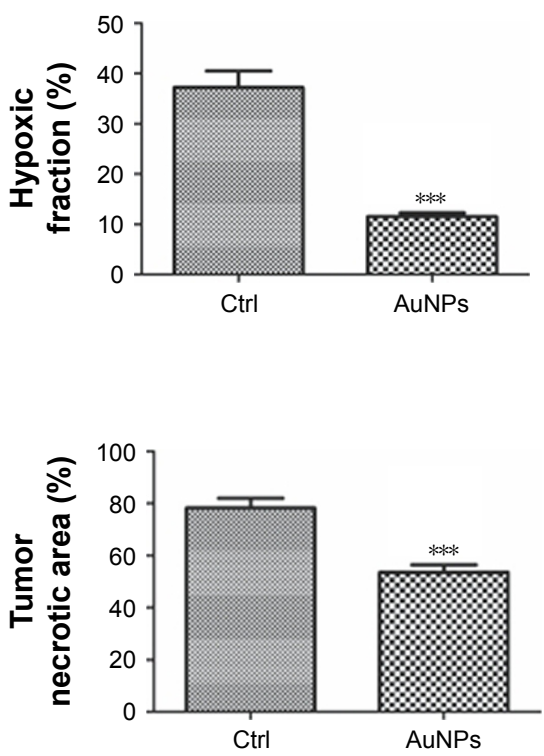

$\mathbf{F}$

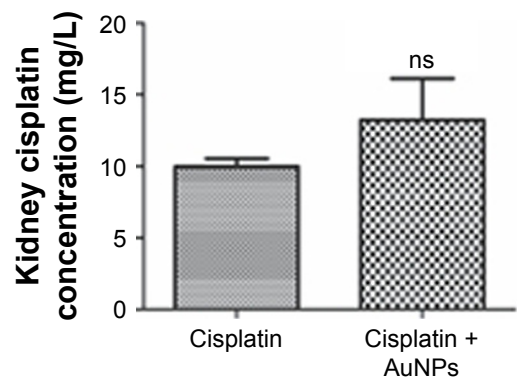

Figure 6 AuNPs increased vascular perfusion as well as drug delivery and reduced tumor hypoxia in SW620 tumor.

Notes: (A) Representative pictures of perfused tumor vessels (green) under immunofluorescence microscopy. The density of perfused vessels was elevated by the administration of AuNPs in SW620 colorectal cancer models ( $n=6, * * P=0.0026)$. Scale bar, $100 \mu \mathrm{m}$. (B) Pimonidazole injection and staining demonstrated that the hypoxic fraction was decreased by the administration of AuNPs in SW620 tumor $(n=6, * * * P<0.00 I)$ due to the increased oxygen supply. Scale bar, I00 $\mu$ m. (C) Necrotic areas in the tumoral regions measured by H\&E staining following treatment with AuNPs. AuNPs reduce necrotic areas in SW620 tumors ( $\mathrm{n}=6$, $* * * P<0.00 \mathrm{I}$ ). Scale bar: I00 $\mu \mathrm{m}$. Cisplatin delivery to the tumor (D), liver (E), and kidney (F) after treatment with AuNPs. AuNPs promoted the cisplatin accumulation in SW620 colorectal cancer models $(n=3$, $* * P=0.0023)$, which, however, did not affect cisplatin accumulation in the liver ( $=3, P=0.2365)$ or the kidney $(n=3, P=0.33 \mid 4)$. Student's $t$-test was utilized for analysis, and error bars indicate SEM.

Abbreviations: AuNPs, gold nanoparticles; Ctrl, control; SEM, standard error of the mean.

and mechanical interplays between cancer cells and stromal cells. ${ }^{31,32}$ In consideration of the collagen density in breast cancers and fibrosis in hepatic cancer as independent risk factors, dense ECM and fibrosis are likely to be involved in tumor initiation. ${ }^{33,34}$ Moreover, the content, organization, and biomechanical properties of the ECM contribute to tumor progression. Like collagen, hyaluronan has prognostic value. There is an association between fibrillar collagen 
A

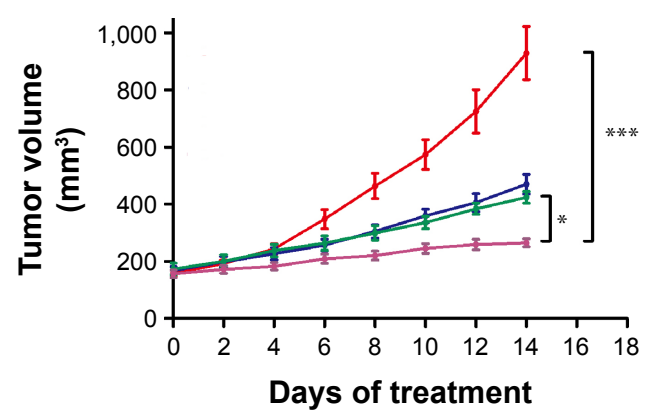

B

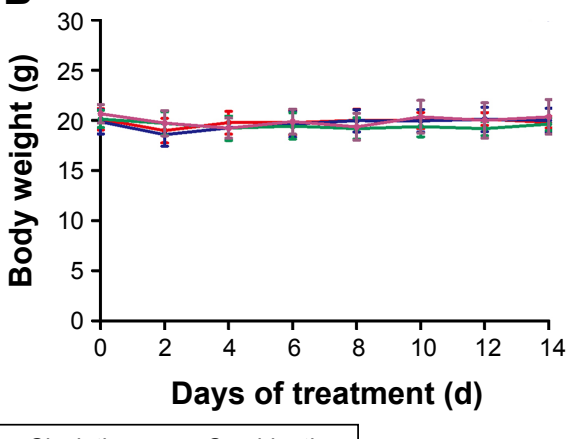

$\rightarrow$ Ctrl $\rightarrow$ AuNPs $\rightarrow$ Cisplatin $\rightarrow$ Combination

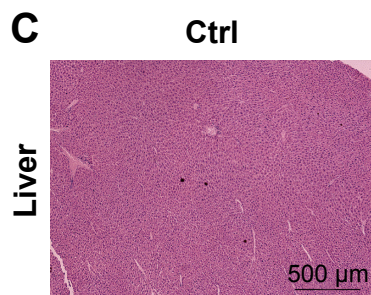

AuNPs

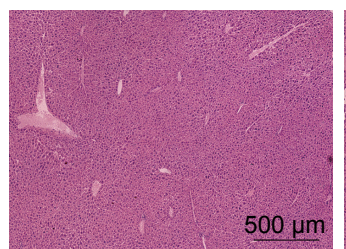

Cisplatin

Combination
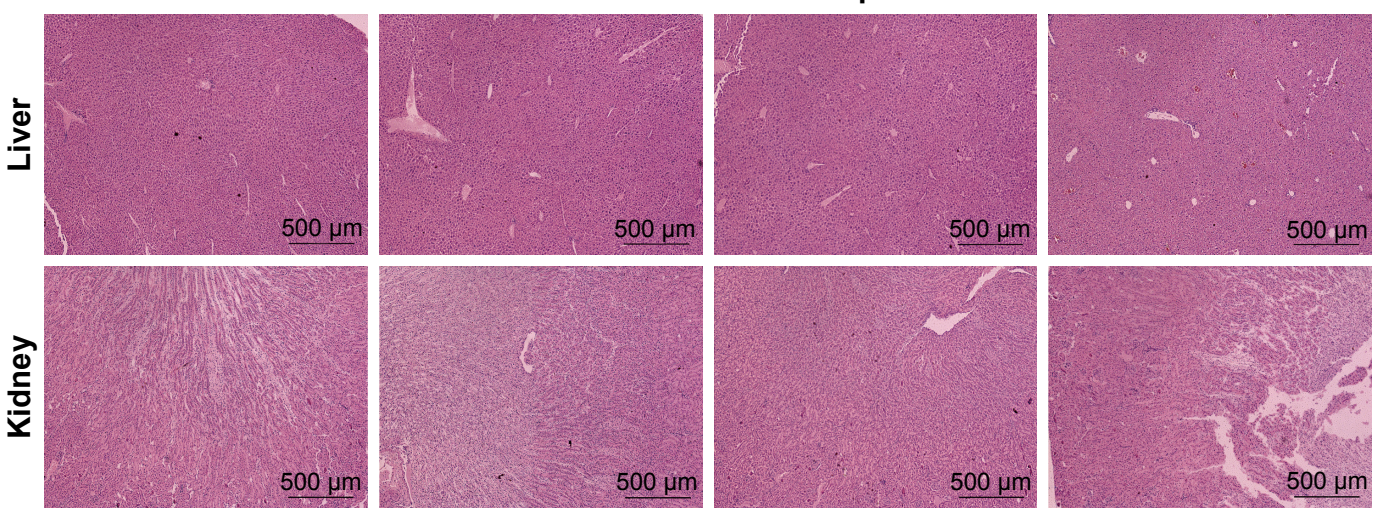

D
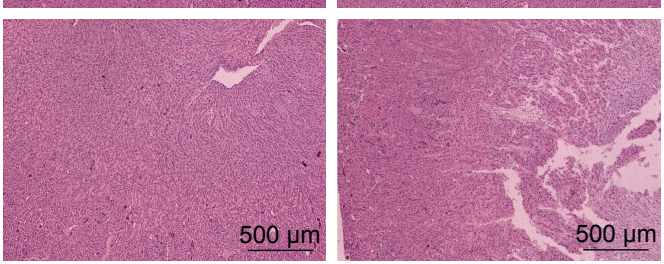

E
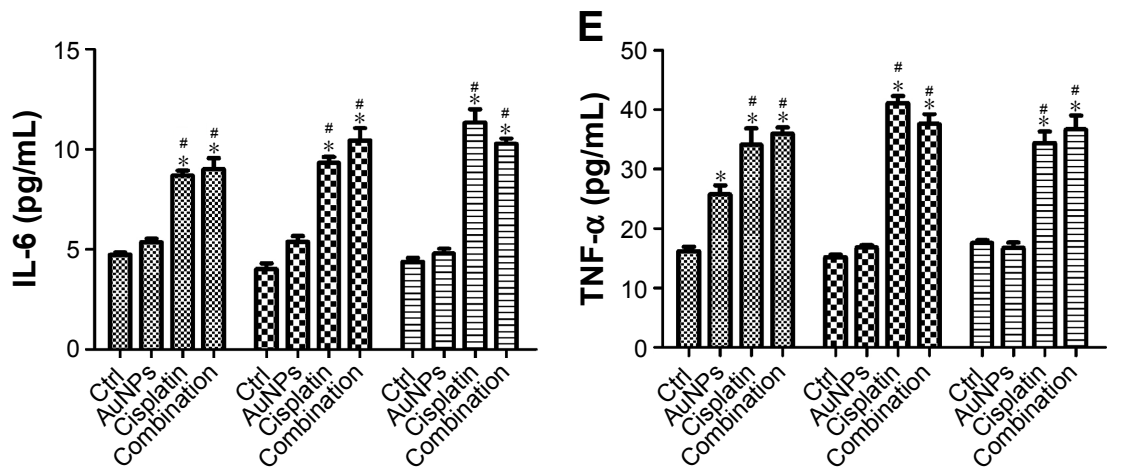

Day 1 Day 5 Day 10

Figure 7 AuNPs potentiated cisplatin efficacy.

Notes: (A) Quantification of tumor volume at different times in mice models treated with AuNPs, cisplatin, combination of AuNPs and cisplatin, or saline. Both cisplatin and AuNPs monotherapy significantly inhibited tumor growth compared with the control treatment, and tumor growth was more greatly inhibited by a combined treatment of cisplatin and AuNPs $(n=6)$. (B) Mouse body weight of the four groups was monitored before and after treatment in different times. (C) Representative images of liver and kidney were removed from the four groups. (D-E) The effect of AuNPs, cisplatin, and combination treatment on the levels of serum IL-6 and TNF- $\alpha$ of mice. The serum IL-6 and TNF- $\alpha$ levels in the cisplatin and combination group were significantly increased comparing with the control and AuNPs groups at day I, day 5 , and day 10 (*compared with the control group, $P<0.05$; "compared with the AuNPs group, $P<0.05)$. One-way ANOVA was utilized for analysis, and error bars indicate $S E M(* P<0.05$, $* * * P<0.00 \mathrm{I})$.

Abbreviations: AuNPs, gold nanoparticles; Ctrl, control; SEM, standard error of the mean.

alignment and poor clinical outcomes in breast cancer. ${ }^{35,36}$ Consistently, elongated collagen fibers have been reported to correlate with unsatisfactory outcomes in patients with colorectal, head and neck, and esophageal cancers. ${ }^{37}$ In addition, the stiffening and organization of the collagen fibers by CAFs have been confirmed to facilitate cancer cell invasion. ${ }^{38,39}$ In spite of the impediment in larger drug distribution by steric interplays with collagen, the delivery of smaller chemotherapy agents is not directly blocked, ${ }^{40}$ which, however, is indirectly hindered by vessel compression. The contribution to solid stress in cancers is observed in collagen as well as hyaluronan, of which, collagen can resist tension, leading to a confined tumor microenvironment. ${ }^{3}$ The attempt to enlarge tumor microenvironment regions 
A

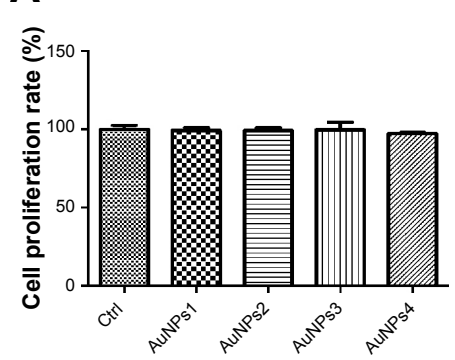

C

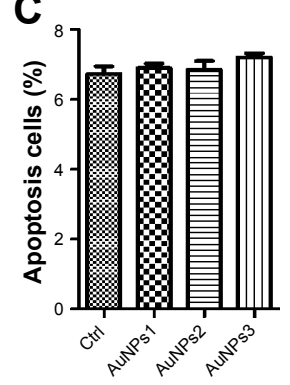

B

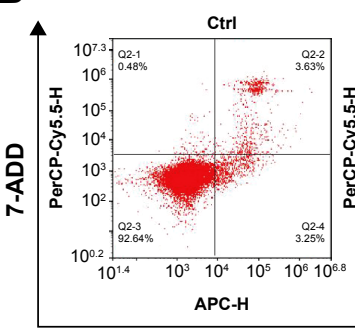

D

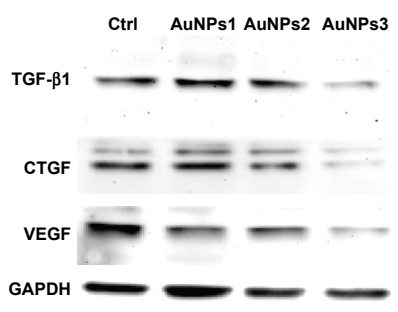

$\mathbf{G}$

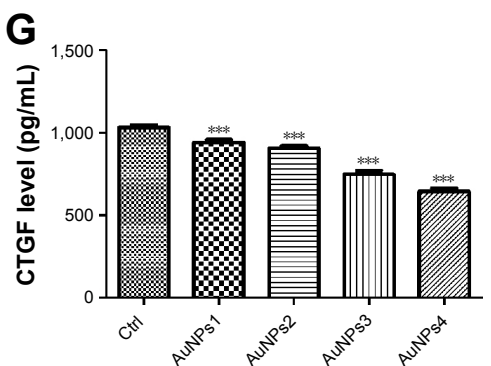

E
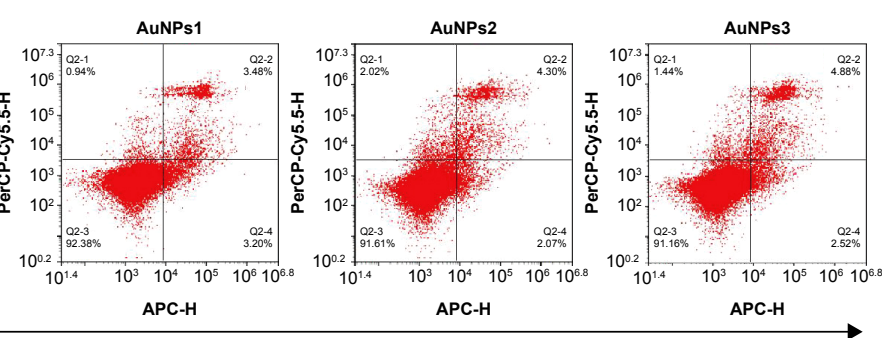

Annexin V-APC
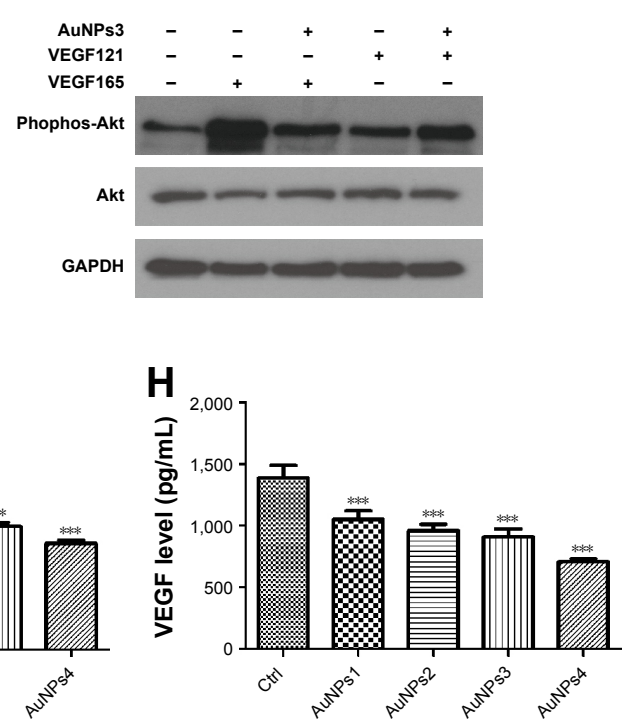

Figure 8 AuNPs reduced TGF- $\beta$ I, CTGF, and VEGF production via Akt-dependent pathway.

Notes: (A) Cell proliferation rate after AuNP treatment with different concentrations was detected by CCK-8 assay $(n=6)$. (B, C) The effect of different treatments on the apoptosis of SW620 cells, no significant difference of the apoptosis rate was found after AuNP treatment with different concentrations ( $\mathrm{n}=3$ ). (D) The effects of AuNPs with different concentrations on TGF- $\beta$ I, CTGF, and VEGF expression of SW620 cells were determined using Western blot analysis. (E) The effects of AuNPs on the expression of Akt and phosphorylated Akt were detected by Western blot analysis. (F-H) The effects of AuNPs with different concentrations on TGF- $\beta$ I, CTGF, and VEGF secretion of SW620 cells were determined using ELISA analysis ( $\mathrm{n}=3$; AuNPs I: $10 \mathrm{ng} / \mathrm{mL}$; AuNPs2: 25 ng/mL; AuNPs3: 50 ng/mL; AuNPs4: I00 ng/mL). One-way ANOVA was utilized for analysis, and error bars indicate SEM (***P<0.00I vs Ctrl group).

Abbreviations: AuNPs, gold nanoparticles; CTGF, connective tissue growth factor; Ctrl, control; SEM, standard error of the mean; VEGF, vascular endothelial growth factor.

during the proliferation of cancer as well as stromal cells persistently exhibits tensile stress, which in turn resists this enlargement through storage of tensile elastic "strain" energy as well as stiffen from the extending collagen fibers. In this way, the cells are confined, contributing to compressive stress arising from the force of active proliferation. Beyond this maximal compression, the excess compressive stress is transmitted to tumor vessels. The newly generated vessels are generally burdened with abnormal structure and dysfunctions, which render poor perfusion as well as oxygenation. In addition, insufficient vascular perfusion confines the delivery of therapeutic agents into the tumor, leading to unsatisfactory chemo/radiotherapy effects, which promotes tumor invasion as well as metastasis. ${ }^{41}$ Therefore, the compressive stress could be lowered by reducing collagen levels in tumor microenvironment, which leads to easy expansion of proliferating cells.

AuNPs, an perfect nano-drug supporter due to its biocompatibility as well as large surface area-to-volume ratio, have been successfully utilized in the delivery of large biomacromolecules without affecting their activity. ${ }^{42}$ The delivery of nanoparticles relies on heterogeneous vessels, due to the passive targeting nature of the process. ${ }^{43}$ The permeability of the nanoparticles and macromolecules into the majority of normal tissues is prohibited by the integrity of the vascular endothelial; therefore, the selective delivery into cancer tissues has the advantage of a long circulation period but less toxicity. In our recent study, AuNPs were reported to be involved in conjunction with recombinant human endostatin, transient indication of tumor vasculature 
A

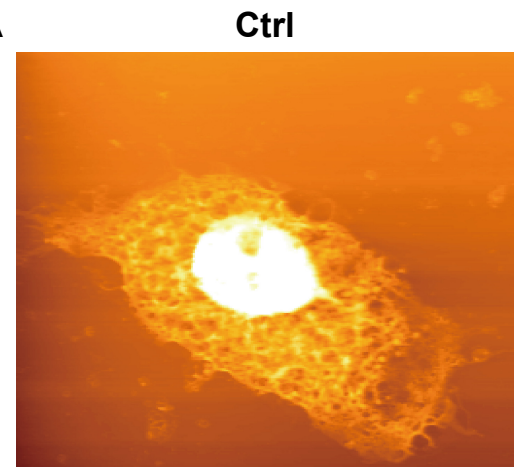

B

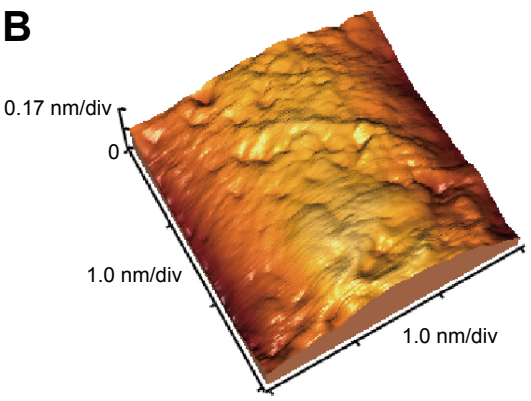

AuNPs1
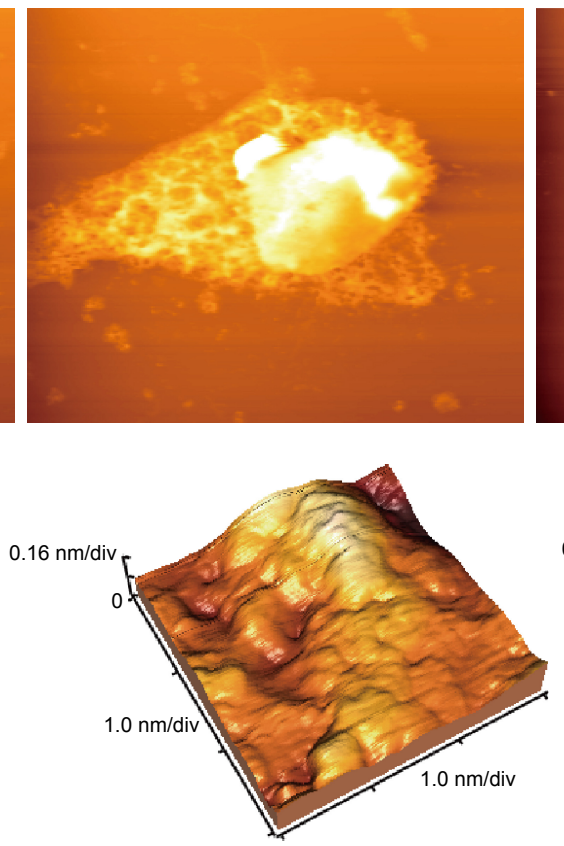

AuNPs2
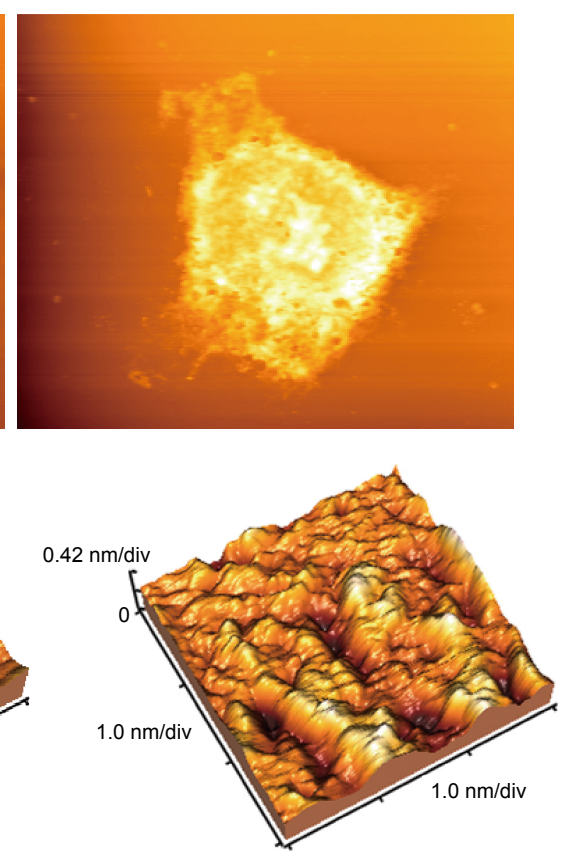

Figure 9 AFM images demonstrate the uptake of AuNPs by SW620 cells.

Notes: (A) Representative morphology images of SW620 cells of the control group and AuNPs group. (B) Representative cellular surface ultrastructure images of SW620 cells (AuNPsl: $10 \mathrm{ng} / \mathrm{mL}$; AuNPs2: $25 \mathrm{ng} / \mathrm{mL}$ ).

Abbreviations: AFM, atomic force microscope; AuNPs, gold nanoparticles; Ctrl, control.

normalization, promotion of tumor-selective delivery, and reversal of epithelial-mesenchymal transition, subsequently, enhancing vessel perfusion and oxygen support in tumors to obtain optimal therapeutic outcomes. ${ }^{12,13}$

In this study, the rapid reduction in tumor stromal collagen I level by AuNPs indicated that collagen I was degraded or destabilized by AuNPs via an undefined mechanism besides blocking the matrix production through TGF- $\beta 1$ and CTGF. TGF- $\beta 1$ and CTGF are the central factors of tissue remodeling and fibrosis, ${ }^{17}$ and the plasma TGF- $\beta 1$ and CTGF were reduced after the treatment with AuNPs, which means that AuNPs decompress stroma in tumor via regulating TGF- $\beta 1$ and CTGF. Regulating the VEGF expression can lead to the normalization of blood vessels, ${ }^{20}$ which help to reduce the permeability of vessels in tumor. Our further in vitro assays also confirmed the mechanism of effects of AuNPs for reduction in TGF- $\beta 1$, CTGF, and VEGF in colorectal cancer cells. The heparin-binding capacity of AuNPs ${ }^{14}$ inhibited VEGF165-induced activation of VEGFR2 and the downstream Akt signaling pathways in SW620 cells. Our results revealed that AuNPs mainly disrupted the VEGF165VEGFR2 interaction, but not VEGF121-VEGFR2 interaction, which is consistent to previous study that AuNPs do not inhibit the activity of non-heparin-binding growth factors such as VEGF121. ${ }^{44}$ Although our results also suggest that AuNPs may mildly enhance the VEGF121-induced AKT phosphorylation. In general, VEGF165 is overexpressed in tumors at a high frequency, and the AuNPs may integrally reduce the AKT phosphorylation in tumor environment. Thus, AuNPs may reduce the production and secretion of TGF- $\beta 1$, CTGF, and VEGF via AKT phosphorylation pathway. However, the underlying mechanism still remains unclear and requires further study. Nevertheless, the AuNPs cannot inhibit SW620 cell viability as previously reported. ${ }^{45}$ Although AuNPs alone have been shown to exert no impact on tumor growth in various studies, especially in photothermal therapy field; ${ }^{46}$ however, different sizes of AuNPs show different effects in VEGF165-induced proliferation of HUVECs, the specific inhibitory effects of AuNPs size are better in 10-20 $\mathrm{nm}$ (diameter). ${ }^{47}$ In our study, the nanosphere of AuNPs and the size of AuNPs used was $15 \mathrm{~nm}$ in our experiment, which was an effective size to inhibit the VEGF165-induced activation. Therefore, the AuNPs inhibit colorectal tumor growth in mice model mainly via reduction of stromal formation in tumor microenvironment. In addition, the decompression of stroma and vessel normalization in tumor may result in the possibility of enhancing drug delivery.

CAF is one of the most important parts of the tumor microenvironment; we found that AuNPs also reduced the density of activated CAFs in colorectal cancer models. Therefore, AuNPs are likely to inhibit the activation of fibroblasts to $\alpha$-SMA + CAF phenotype, thereby resulting in reduced 
CAF density. Altogether, these combined reactions caused reduced levels of collagen I in colorectal cancer models. Our results have confirmed that AuNPs reduced solid stress in colorectal cancer, indicating that AuNPs are likely to decompress vessels in desmoplastic tumors by relieving solid stress due to its antimatrix roles. In addition, our results also demonstrate that AuNPs increases tumor vessel perfusion through the reduction in solid stress in our models. In consideration of the governing role of vessel perfusion in oxygen as well as drug delivery, pimonidazole staining was used to determine tumor oxygenation. Consequently, oxygen concentrations were maintained in SW620 tumors after the administration of AuNPs, while a typical growth-dependent decline in oxygenation was observed in the control group. Therefore, reduction in tumor solid stress via "microenvironmental normalization" mediated by AuNPs promoted the oxygen delivery to cancers. HPLC analysis was employed in the examination of the concentration of cisplatin in tumors, which was increased by the administration of AuNPs mainly due to the elevated blood flow in tumors. Moreover, although previous study ${ }^{48}$ reported that the most important factor of cisplatin resistance in cancers is the reduction in drug accumulation in the tumor or in the cancer cell, enhancing the drug delivery by combined use of AuNPs could be considered as one of the potential strategies to overcome the resistance. Our results also further indicated that AuNPs cannot significantly increase the toxicity when combined with cisplatin. These data implicate that enhancing cisplatin delivery by AuNPs could be a safe and potent strategy for colorectal cancer.

In summary, despite the previous classification of AuNPs as antiangiogenics, ${ }^{14}$ in this study, we demonstrated that AuNPs can enhance cisplatin delivery by vascular decompression attributed to the decreased stromal activity as well as confined production of stroma components which caused compression. Remarkably, AuNPs targeted both CAFs and collagen, two recognized contributors to solid stress. ${ }^{3}$ In addition, we suggested that inhibitors of downstream Akt signaling through TGF- $\beta 1$, CTGF, or VEGF might exert similar effects in decreasing solid stress to sensitize chemotherapy. Undoubtedly, AuNPs are a potential strategy in colorectal cancer therapies for combined with using cisplatin.

\section{Conclusion}

In this study, we verified that AuNPs decreased the colorectal CAF density, reduced the production of tumor stromal collagen $\mathrm{I}$, and diminished the expression of profibrotic signals, including CTGF, TGF- $\beta 1$, and VEGF. Consequently, AuNPs could alleviate solid stress in tumors, leading to enhanced vessel perfusion. Subsequently, cisplatin and oxygen delivery to tumors were improved by AuNPs, which reduced hypoxia while sensitizing chemotherapy in colorectal cancer model. Hence, AuNPs were effective agents in enhancing cisplatin delivery and inhibiting tumor growth by decompressing colorectal cancer vessels.

\section{Availability of data and materials}

All the data and materials are fully available without restriction.

\section{Acknowledgment}

This study was supported by the National Natural Science Foundation of China (81472849), the Guangdong Natural Science Research (2014A030313383), and the Guangdong High-level University Construction Fund for Jinan University (88016013034).

\section{Author contributions}

YP conceived the experiments. XZ, WL, and JP performed the studies and statistical analyses, interpreted the data, and wrote the manuscript. WY collected the materials and managed references. XZ, LQ, and YP conducted the experiments. All the authors reviewed the manuscript. All authors contributed toward data analysis, drafting and revising the paper and agree to be accountable for all aspects of the work.

\section{Disclosure}

The authors report no conflicts of interest in this work.

\section{References}

1. Chauhan VP, Stylianopoulos T, Boucher Y, Jain RK. Delivery of molecular and nanoscale medicine to tumors: transport barriers and strategies. Annu Rev Chem Biomol Eng. 2011;2:281-298.

2. Jain RK. Normalizing tumor microenvironment to treat cancer: bench to bedside to biomarkers. J Clin Oncol. 2013;31(17):2205-2218.

3. Stylianopoulos T, Martin JD, Chauhan VP, et al. Causes, consequences, and remedies for growth-induced solid stress in murine and human tumors. Proc Natl Acad Sci U S A. 2012;109(38):15101-15108.

4. Stylianopoulos T, Martin JD, Snuderl M, Mpekris F, Jain SR, Jain RK. Coevolution of solid stress and interstitial fluid pressure in tumors during progression: implications for vascular collapse. Cancer Res. 2013;73(13): 3833-3841.

5. Janmey PA, McCulloch CA. Cell mechanics: integrating cell responses to mechanical stimuli. Annu Rev Biomed Eng. 2007;9:1-34.

6. Dasari S, Tchounwou PB. Cisplatin in cancer therapy: molecular mechanisms of action. Eur J Pharmacol. 2014;740:364-378.

7. Pathak RK, Wen R, Kolishetti N, Dhar S. A Prodrug of Two Approved Drugs, Cisplatin and Chlorambucil, for Chemo War Against Cancer. Mol Cancer Ther. 2017;16(4):625-636.

8. Sorensen AG, Emblem KE, Polaskova $P$, et al. Increased survival of glioblastoma patients who respond to antiangiogenic therapy with elevated blood perfusion. Cancer Res. 2012;72(2):402-407.

9. Whatcott CJ, Diep CH, Jiang P, et al. Desmoplasia in Primary Tumors and Metastatic Lesions of Pancreatic Cancer. Clin Cancer Res. 2015;21(15): 3561-3568. 
10. Öhlund D, Elyada E, Tuveson D. Fibroblast heterogeneity in the cancer wound. J Exp Med. 2014;211(8):1503-1523.

11. Maeda H, Nakamura H, Fang J. The EPR effect for macromolecular drug delivery to solid tumors: Improvement of tumor uptake, lowering of systemic toxicity, and distinct tumor imaging in vivo. Adv Drug Deliv Rev. 2013;65(1):71-79.

12. Li W, Zhao X, Du B, et al. Gold Nanoparticle-Mediated Targeted Delivery of Recombinant Human Endostatin Normalizes Tumour Vasculature and Improves Cancer Therapy. Sci Rep. 2016;6:30619.

13. Li W, Li X, Liu S, et al. Gold nanoparticles attenuate metastasis by tumor vasculature normalization and epithelial-mesenchymal transition inhibition. Int J Nanomedicine. 2017;12:3509-3520.

14. Pan Y, Ding H, Qin L, Zhao X, Cai J, Du B. Gold nanoparticles induce nanostructural reorganization of VEGFR2 to repress angiogenesis. J Biomed Nanotechnol. 2013;9(10):1746-1756.

15. Pan Y, Wu Q, Liu R, et al. Inhibition effects of gold nanoparticles on proliferation and migration in hepatic carcinoma-conditioned HUVECs. Bioorg Med Chem Lett. 2014;24(2):679-684.

16. Pan Y, Wu Q, Qin L, Cai J, Du B. Gold nanoparticles inhibit VEGF165induced migration and tube formation of endothelial cells via the Akt pathway. Biomed Res Int. 2014;2014:418624.

17. Mori T, Kawara S, Shinozaki M, et al. Role and interaction of connective tissue growth factor with transforming growth factor-beta in persistent fibrosis: A mouse fibrosis model. J Cell Physiol. 1999;181(1): 153-159.

18. Papageorgis $\mathrm{P}$, Stylianopoulos T. Role of TGF $\beta$ in regulation of the tumor microenvironment and drug delivery (review). Int J Oncol. 2015; 46(3):933-943.

19. Siveen KS, Prabhu K, Krishnankutty R, et al. Vascular Endothelial Growth Factor (VEGF) Signaling in Tumour Vascularization: Potential and Challenges. Curr Vasc Pharmacol. 2017;15(4):339-351.

20. Grothey A. VEGF inhibition beyond tumour progression. Lancet Oncol. 2013;14(1):2-3

21. Danese S. Inflammation and the mucosal microcirculation in inflammatory bowel disease: the ebb and flow. Curr Opin Gastroenterol. 2007; 23(4):384-389.

22. Maruotti N, Cantatore FP, Crivellato E, Vacca A, Ribatti D. Angiogenesis in rheumatoid arthritis. Histol Histopathol. 2006;21(5):557-566.

23. Özdemir BC, Pentcheva-Hoang T, Carstens JL, et al. Depletion of carcinoma-associated fibroblasts and fibrosis induces immunosuppression and accelerates pancreas cancer with reduced survival. Cancer Cell. 2014;25(6):719-734.

24. Kalluri R, Zeisberg M. Fibroblasts in cancer. Nat Rev Cancer. 2006; 6(5):392-401.

25. Augsten M. Cancer-associated fibroblasts as another polarized cell type of the tumor microenvironment. Front Oncol. 2014;4:62.

26. Zlotek-Zlotkiewicz E, Monnier S, Cappello G, Le Berre M, Piel M. Optical volume and mass measurements show that mammalian cells swell during mitosis. J Cell Biol. 2015;211(4):765-774.

27. Orimo A, Gupta PB, Sgroi DC, et al. Stromal fibroblasts present in invasive human breast carcinomas promote tumor growth and angiogenesis through elevated SDF-1/CXCL12 secretion. Cell. 2005;121(3): 335-348.

28. Li ZD, Bork JP, Krueger B, et al. VEGF induces proliferation, migration, and TGF-beta1 expression in mouse glomerular endothelial cells via mitogen-activated protein kinase and phosphatidylinositol 3-kinase. Biochem Biophys Res Commun. 2005;334(4):1049-1060.

29. Suzuma K, Naruse K, Suzuma I, et al. Vascular endothelial growth factor induces expression of connective tissue growth factor via KDR, Flt1, and phosphatidylinositol 3-kinase-akt-dependent pathways in retinal vascular cells. J Biol Chem. 2000;275(52):40725-40731.
30. Adya R, Tan BK, Punn A, Chen J, Randeva HS. Visfatin induces human endothelial VEGF and MMP-2/9 production via MAPK and PI3K/Akt signalling pathways: novel insights into visfatin-induced angiogenesis. Cardiovasc Res. 2008;78(2):356-365.

31. Lu P, Weaver VM, Werb Z. The extracellular matrix: a dynamic niche in cancer progression. J Cell Biol. 2012;196(4):395-406.

32. Tung JC, Barnes JM, Desai SR, et al. Tumor mechanics and metabolic dysfunction. Free Radic Biol Med. 2015;79:269-280.

33. Boyd NF, Guo H, Martin LJ, et al. Mammographic density and the risk and detection of breast cancer. N Engl J Med. 2007;356(3):227-236.

34. Elsharkawy AM, Mann DA. Nuclear factor-kappaB and the hepatic inflammation-fibrosis-cancer axis. Hepatology. 2007;46(2):590-597.

35. Conklin MW, Eickhoff JC, Riching KM, et al. Aligned collagen is a prognostic signature for survival in human breast carcinoma. Am J Pathol. 2011;178(3):1221-1232.

36. Bredfeldt JS, Liu Y, Conklin MW, Keely PJ, Mackie TR, Eliceiri KW. Automated quantification of aligned collagen for human breast carcinoma prognosis. J Pathol Inform. 2014;5(1):28.

37. Hanley CJ, Noble F, Ward M, et al. A subset of myofibroblastic cancer-associated fibroblasts regulate collagen fiber elongation, which is prognostic in multiple cancers. Oncotarget. 2016;7(5):6159-6174.

38. Gaggioli C, Hooper S, Hidalgo-Carcedo C, et al. Fibroblast-led collective invasion of carcinoma cells with differing roles for RhoGTPases in leading and following cells. Nat Cell Biol. 2007;9(12):1392-1400.

39. Goetz JG, Minguet S, Navarro-Lérida I, et al. Biomechanical remodeling of the microenvironment by stromal caveolin-1 favors tumor invasion and metastasis. Cell. 2011;146(1):148-163.

40. Pluen A, Boucher Y, Ramanujan S, et al. Role of tumor-host interactions in interstitial diffusion of macromolecules: cranial vs. subcutaneous tumors. Proc Natl Acad Sci U S A. 2001;98(8):4628-4633.

41. Liu WB, Wang XP, Wu K, Zhang RL. Effects of angiotensin II receptor antagonist, Losartan on the apoptosis, proliferation and migration of the human pancreatic stellate cells. World J Gastroenterol. 2005; 11(41):6489-6494.

42. Mitra P, Chakraborty PK, Saha P, Ray P, Basu S. Antibacterial efficacy of acridine derivatives conjugated with gold nanoparticles. Int J Pharm. 2014;473(1-2):636-643.

43. Behr TM, Memtsoudis S, Sharkey RM, et al. Experimental studies on the role of antibody fragments in cancer radio-immunotherapy: Influence of radiation dose and dose rate on toxicity and anti-tumor efficacy. Int J Cancer. 1998;77(5):787-795.

44. Mukherjee P, Bhattacharya R, Wang P, et al. Antiangiogenic properties of gold nanoparticles. Clin Cancer Res. 2005;11(9):3530-3534.

45. Arnida, Malugin A, Ghandehari H. Cellular uptake and toxicity of gold nanoparticles in prostate cancer cells: a comparative study of rods and spheres. J Appl Toxicol. 2010;30(3):212-217.

46. Bucharskaya A, Maslyakova G, Terentyuk G, et al. Towards Effective Photothermal/Photodynamic Treatment Using Plasmonic Gold Nanoparticles. Int J Mol Sci. 2016;17(8):E1295.

47. Arvizo RR, Rana S, Miranda OR, Bhattacharya R, Rotello VM, Mukherjee P. Mechanism of anti-angiogenic property of gold nanoparticles: role of nanoparticle size and surface charge. Nanomedicine. 2011; 7(5):580-587.

48. Loh SY, Mistry P, Kelland LR, Abel G, Harrap KR. Reduced drug accumulation as a major mechanism of acquired resistance to cisplatin in a human ovarian carcinoma cell line: circumvention studies using novel platinum (II) and (IV) ammine/amine complexes. Br J Cancer. 1992;66(6): 1109-1115. 
International Journal of Nanomedicine

Dovepress

\section{Publish your work in this journal}

The International Journal of Nanomedicine is an international, peerreviewed journal focusing on the application of nanotechnology in diagnostics, therapeutics, and drug delivery systems throughout the biomedical field. This journal is indexed on PubMed Central, MedLine, CAS, SciSearch ${ }^{\circledR}$, Current Contents ${ }^{\circledR} /$ Clinical Medicine,
Journal Citation Reports/Science Edition, EMBase, Scopus and the Elsevier Bibliographic databases. The manuscript management system is completely online and includes a very quick and fair peer-review system, which is all easy to use. Visit http://www.dovepress.com/ testimonials.php to read real quotes from published authors.

Submit your manuscript here: http://www.dovepress.com/international-journal-of-nanomedicine-journal 